\title{
ESTUDO DE PROJEÇÕES DE UM BOF UTILIZANDO ANÁLISE MULTIVARIADA DE DADOS
}

\author{
Bruna Helena Malovini Loiola ' \\ Henrique Silva Furtado ${ }^{2}$ \\ André Vicente Salazar ${ }^{3}$ \\ Itavahn Alves da Silva ${ }^{4}$ \\ Carlos Antônio da Silva '
}

\begin{abstract}
Resumo
A projeção em um BOF é uma anomalia que representa desperdício de matéria prima, risco de acidentes, prejuízo financeiro e, principalmente, traz consequências ambientais. Devido à relevância deste fato foram realizados acompanhamentos in loco na ArcelorMittal Tubarão (AMT), e arquivos de Big Data advindos da operação de sopro de oxigênio dos convertedores foram analisados com o intuito de investigar as principais variáveis associadas ao fenômeno de projeção. Realizou-se o tratamento descritivo, por meio das técnicas de Análise de Cluster, Análise de Fatores, e de Componentes Principais com a finalidade de elaborar uma equação de regressão linear multivariada que pudesse modelar e prever as corridas com projeções. O tratamento estatístico foi feito com a utilização do software Statistica e do NCSS Statistical Software. A análise conclui que as variáveis teor de silício no gusa líquido, peso de minério adicionado, peso de cal calcítica e tempo de sopro de oxigênio estão entre as principais causas de projeção.
\end{abstract}

Palavras-chave: Projeção de escória; BOF; Análise multivariada; Big Data.

\section{STUDY OF SLOPPING IN A BOF USING MULTIVARIATE ANALYSIS DATA}

\begin{abstract}
Slopping in a BOF is an anomaly that leads to waste of raw material, accident hazard, financial losses, and mainly, brings environmental consequences. Due to its relevance on-site monitoring was performed at ArcelorMittal Tubarão (AMT), and Big Data files were analyzed with the purpose of investigating the main variables associated with slopping. The descriptive treatment was carried out using Cluster Analysis, Factor Analysis, and Principal Components to elaborate a multivariate linear regression equation capable of forecasting slopping. The statistical treatment was performed with the support of the Statistica and NCSS Statistical Software. According with this analysis the hot metal Silicon content, the weight of iron ore added as a coolant, the weight of calcined lime and the oxygen blowing time are among the main causes of slopping.
\end{abstract}

Keywords: Slopping; BOF; Multivariate analysis; Big data.

\section{INTRODUÇÃO}

A função do processo BOF (Basic Oxygen Furnace) é produzir um aço base reduzindo as impurezas da carga metálica através de oxidação, principalmente do carbono e do silício, além da adequação dos teores de fósforo. Como resultado, ocorre uma grande geração de gases oriundos da oxidação do carbono e a formação de uma escória líquida decorrente da reação do produto da oxidação das demais impurezas com materiais fluxantes adicionados para estabilizar tais reações [I]. A combinação da grande evolução gasosa com o desenvolvimento da escória (volume e química), segundo Fruehan and Turkdogan [2], Albani et al. [3], Hewage [4], e Harada et al. [5], leva à formação de uma emulsão. Se por um lado a emulsão acelera as reações de refino, por outro, se não controlada, pode transbordar de forma violenta para a área externa do convertedor. Esta projeção de metal e escória representa uma anomalia em

'Rede Temática em Engenharia de Materiais - REDEMAT, Universidade Federal de Ouro Preto - UFOP, Ouro Preto, MG, Brasil. E-mail: malovinibruna@gmail.com ${ }^{2}$ Centro de Pesquisa, ArcelorMittal, Vitória, ES, Brasil.

${ }^{3}$ Instituto Federal do Espírito Santo - IFES, Vitória, ES, Brasil.

${ }^{4}$ Universidade Federal de Ouro Preto - UFOP, Ouro Preto, MG, Brasil.

2176-1523 (c) 2020 Associação Brasileira de Metalurgia, Materiais e Mineração. Publicado pela ABM. Este é um artigo de acesso aberto distribuído sob os termos da licença Creative Commons CC BY-NC-ND (Attribution-NonCommercial-NoDerivs) - https:// creativecommons.org/licenses/by-nc-nd/4.0\%. 
convertedores de aciaria, sendo que o controle da mesma é importante, uma vez que o sistema de captação de gases do convertedor, em geral, não suporta o excesso de gases que repentinamente são expelidos no momento da projeção [6]. A consequência é que acabam sendo eliminados gases em forma de poluente ao ambiente externo à aciaria. Além disso, com a projeção há um risco de ocorrência de acidentes, e também se reduz o rendimento de produção de aço porque há perdas metálicas, uma vez que cerca de 10 a $20 \%$ da quantidade total de massa metálica é suspensa na emulsão [7]. Dadas estas consequências, métodos de detecção e controle de projeção foram propostos, como citados em Walker et al. [8], Harada et al. [5], e Carvalho et al. [9]. Dessa forma, a proposta desse trabalho é utilizar métodos de análises multivariadas, ainda pouco comuns na área de siderurgia, para efetuar uma análise de Big Data de tal forma a detectar as variáveis de influência nas ocorrências de projeções em um convertedor da ArcelorMittal Tubarão, além da elaboração de um modelo matemático que possa prever as corridas com projeção. Para este estudo foram utilizadas técnicas tais como Análises de Cluster, de Análise de Fatores (AF), Análise de Componentes Principais (ACP), e análises de Regressões Múltiplas e de Componentes Principais.

\section{MATERIAIS E MÉTODOS}

Para a análise descrita neste trabalho foram utilizados dados obtidos junto à ArcelorMittal Tubarão (Serra, ES - Brasil). Os dados operacionais compreendem o período de maio de 2016 a maio de 2017 e incluem 7735 corridas do convertedor I, com informações referentes a 39 variáveis de interesse. Os dados foram transportados para uma matriz e seus valores normalizados (entre 0 e I). Em seguida, aplicaram-se as técnicas estatísticas de Análise de Cluster, Análise de Fatores (AF) e Analise de Componentes Principais (ACP), utilizando o software Statistica versão 7.0. A Análise de Cluster permite agrupar as variáveis com base em critérios pré-definidos, gerando um dendograma, - qual possibilita a realização de uma análise de afinidade entre as variáveis independentes e destas com a variável dependente. $\mathrm{Na}$ AMT, ao atribuir valores a essa variável resposta, o operador da aciaria insere, com base em sua observação, um valor numérico representando se aconteceu ou não projeção na corrida. A planilha de dados analisada apresenta em duas de suas colunas dados da variável resposta: a primeira (variável 25) avalia se a projeção ocorreu até a primeira metade do sopro e a segunda (variável 26) avalia se a projeção ocorreu da metade até o final do processo. Essas duas variáveis determinam qual agrupamento deve ser selecionado para as etapas seguintes de análise. A técnica estatística da Análise de Fatores (AF) foi aplicada com a finalidade de reduzir o número de variáveis independentes do conjunto de dados a partir da criação de novas variáveis sintéticas, chamadas de fatores; e assim, determinar quantas das novas variáveis obtidas deveriam ser analisadas, bem como determinar qual seria a correlação entre as variáveis originais e os fatores, e quais os índices de contribuição das variáveis originais nos fatores. Por sua vez, a técnica de Análise de Componentes Principais (ACP) foi aplicada para substituir as variáveis por outras sintéticas, o que permite indicar o número ideal de fatores a ser analisado.

As variáveis que mais contribuem para as Componentes Principais (CP) foram consideradas para se obter um modelo que prevê projeção a partir de análises de regressão linear múltipla e de Regressão por Componentes Principais (RCP). As variáveis respostas do Statistica foram utilizadas nesta etapa porque permitem a simplificação da análise, ao excluir as variáveis que não possuem efeitos significativos sobre a projeção. Em consequência, foi utilizada uma matriz formada apenas com os valores de variáveis que mais influenciam a projeção; além do mais, as duas variáveis de projeção foram transformadas em apenas uma, com valores assinalados como 0 para não projeção e I para projeção. $O$ programa NCSS aplicado a esta matriz gerou um relatório sobre a regressão. Primeiro se fez a RCP que avalia dados de regressão múltipla que possuem multicolinearidade. Quando ocorre a multicolinearidade, as estimativas dos mínimos quadrados são imparciais, mas suas variações são grandes, e podem, portanto, estar longe do verdadeiro valor. Assim, a RCP reduz o erro padrão, ou seja, espera-se estimativas mais confiáveis [10]. Em seguida, para que fosse possível uma comparação de resultados, fez-se a regressão múltipla, que analisa o relacionamento entre a variável dependente (projeção ou não projeção) e as variáveis de processo independentes [ I I]. Por fim, o modelo matemático foi testado.

\section{RESULTADOS E DISCUSSÃO}

\section{I Análises no Statistica}

Foram primeiramente selecionadas as 39 variáveis envolvendo o convertedor I. A partir do dendograma produzido pelo software Statistica, decidiu-se por um intercepto próximo de I,6, em destaque na Figura la, de forma a identificar um grupo com número aceitável de variáveis para análise. Cada número da horizontal no diagrama identifica uma variável contida nos dados. Todas as 7 contidas no agrupamento número 3 foram utilizadas na etapa seguinte da análise porque as variáveis de projeção estão melhor conectadas. Assim, com base no uso da ferramenta de análise fatorial, para a redução do número de variáveis, obteve-se a tabela da Figura Ib.

Para as 7 variáveis, o software indicou 7 fatores, porém, nem todas foram consideradas, seguindo-se o objetivo de reduzir o número de variáveis. Para análises estatísticas, considera-se, geralmente, até o fator que apresente porcentagem cumulativa maior que 70, logo, neste caso, até o fator número 4.

$\mathrm{Na}$ Figura Ib, a primeira coluna representa os autovalores de cada fator, a segunda representa a variância 
a)

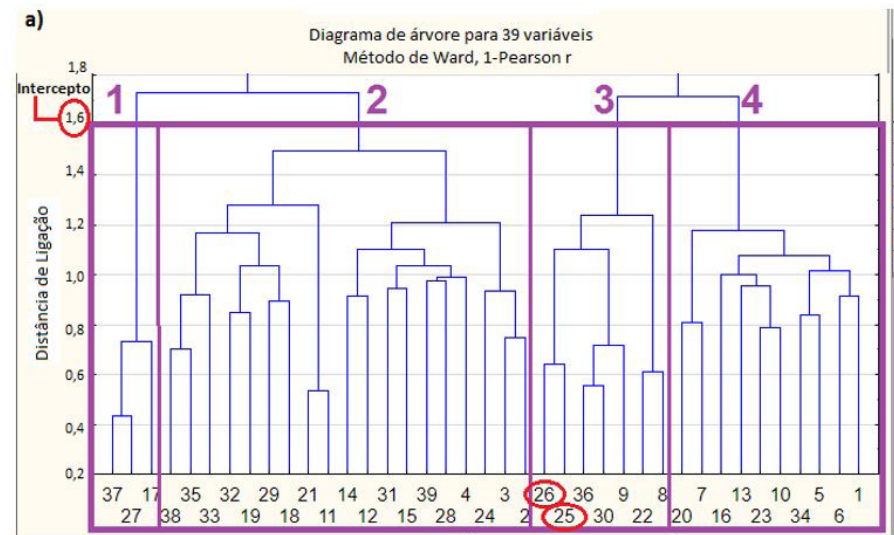

b)

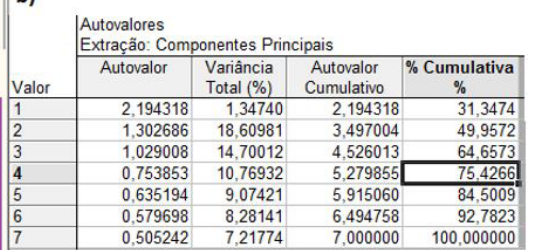

Figura I. (a) Dendograma formado a partir da Análise de Cluster; (b) Quadro gerado com o número de autovalores, variância total, autovalor acumulativo e porcentagem cumulativa para cada autovalor.

Tabela I. Tabela de contribuição das variáveis originais em cada fator

\begin{tabular}{cccc}
\hline Variável & Fator I & Fator 2 & Fator 3 \\
\hline 8 & 0,037918 & 0,971831 & 0,056711 \\
9 & 0,044692 & 0,022098 & 0,121799 \\
22 & 0,009853 & 0,193379 & 0,087873 \\
25 & 0,086824 & 0,037477 & 0,972485 \\
26 & 0,177202 & 0,023608 & 0,083696 \\
30 & 0,087407 & 0,058543 & 0,043500 \\
36 & 0,975468 & 0,117793 & 0,959315 \\
\hline
\end{tabular}

Tabela 2. Variáveis que mais influenciam nas projeções do convertedor I

\begin{tabular}{cccccc}
\hline Variável & Fator & Nome & Variável & Fator & Nome \\
\hline 36 & $\mathrm{I}$ & Si no gusa líquido \% & 30 & 3 & Tempo de sopro de oxigênio min) \\
8 & 2 & Peso de minério $(\mathrm{t})$ & 9 & 4 & Peso de cal calcítica (t) \\
\hline
\end{tabular}

total de cada um, o terceiro e o quarto representam, respectivamente, os autovalores acumulados e a porcentagem cumulativa de cada fator.

De acordo com a análise das CP, o mesmo conteúdo da Figura Ib foi obtido. Assim, foi possível confirmar o número de $\mathrm{CP}$ (fatores) a serem analisados como influências de projeção. A etapa seguinte consistiu do emprego da ferramenta Varimax Normalized, a qual verifica quais variáveis originais mais contribuem para a formação dos fatores. As variáveis selecionadas foram aquelas que possuem valores significativos (maiores que 0,7 ) e que mais contribuem para a formação de cada fator, conforme a Tabela I. Neste caso, cada fator foi explicado por apenas uma variável.

As variáveis de maior contribuição, para cada fator, são apresentadas na Tabela 2 e estão de acordo com o esperado, segundo a literatura.

A presença do Si no gusa líquido está relacionada à projeção porque quanto maior o teor deste elemento, maior o volume de escória. Os resultados de causas também incluem a adição de refrigerante (minério), que aumenta a contribuição de oxigênio para a reação de descarburação na emulsão e causa o aumento da viscosidade da emulsão devido à diminuição da temperatura. Uma maior viscosidade pode levar à maior retenção dos gases na emulsão. Quando o operador detecta a possibilidade de projeção a vazão de oxigênio é reduzida(seja manualmente ou automaticamente) o que se reflete em tempos de sopro mais longos para as mesmas necessidades de oxidação; o que denota que o padrão de sopro original era inadequado. Uma maior adição de cal calcítica pode implicar em acréscimo da viscosidade aparente devido à presença de sólidos dispersos durante o período de dissolução e/ou maior volume de escória, que inibem a liberação dos gases das reações, o que também auxilia no crescimento da emulsão. $O$ uso da Análise de Cluster, $A F$ e ACP não indica a relação causa efeito das variáveis na projeção, o que precisa ser avaliado pelo operador do processo. Entretanto, estas técnicas permitem agrupar as variáveis correlacionadas à projeção, e reduzir o número de variáveis existentes para análise.

\subsection{Regressão Linear no NCSS}

Os resultados da regressão linear geraram um modelo matemático para a previsão de corridas com projeção. O primeiro teste feito foi a partir da RCP. Esta análise gera dados e, automaticamente, os compara com o uso da 
Loiola et al.

Tabela 3. Coeficientes gerados por RCP, Regressão por Mínimos Quadrados (RMQ) e via NCSS

\begin{tabular}{|c|c|c|c|c|c|c|}
\hline Variável & Fator & $\mathrm{Bi}(\mathrm{RCP})$ & Erro & $\mathrm{Bi}(\mathrm{RMQ})$ & Erro & Nome da Variável $\left(X_{i}\right)$ \\
\hline & & $-0,3078$ & & $-0,2970$ & & Intercepto \\
\hline C34 & 1 & 0,7547 & 0,04 & 0,8609 & 0,09 & Si no gusa líquido (\%) \\
\hline $\mathrm{C} 8$ & 2 & 0,0862 & 0,03 & 0,0862 & 0,03 & Peso de minério adicionado $(t)$ \\
\hline C9 & 4 & 0,6648 & 0,06 & 0,6378 & 0,06 & Peso de cal calcítica (t) \\
\hline \multirow[t]{2}{*}{ C28 } & 3 & $0,88 \mid 4$ & 0,09 & 0,7840 & 0,12 & Tempo de sopro de O2 (min) \\
\hline & $r^{2}$ & 0,5959 & & 0,6570 & & \\
\hline
\end{tabular}

Tabela 4. Principais valores encontrados para a variável projeção $\left(M_{p i}\right)$

\begin{tabular}{lcc}
\hline & $\begin{array}{c}\text { Corridas com } \\
\text { projeção }\end{array}$ & $\begin{array}{c}\text { Corridas sem } \\
\text { projeção }\end{array}$ \\
\hline Máximo; Média; Mínimo & 0,$97 ; 0,24 ; 0,16$ & 0,$86 ; 0,12 ;-0,25$ \\
\hline
\end{tabular}

técnica de mínimos quadrados de regressão linear múltipla. As variáveis respostas da Tabela 2 foram utilizadas de forma a gerar um modelo matemático pelo software NCSS. Foram obtidos os dados da Tabela 3. A primeira coluna e a última coluna identificam os fatores e o os nomes das variáveis selecionadas. A terceira coluna identifica o coeficiente de cada variável por RCP e a quinta coluna mostra o coeficiente de cada variável por regressão múltipla. Percebe-se, então, que a linha de regressão linear múltipla está melhor ajustada uma vez que apresenta um valor de $r^{2}$ superior. Tomando a regressão linear múltipla, portanto, como a mais adequada dentre as duas, tem-se o seguinte modelo matemático (I) gerado para previsão de corridas com projeções:

$$
M_{p i}=a+\sum B_{i} X_{i}
$$

Onde $M_{\mathrm{pi}}$ é a variável dependente (com valor de 0 para não projeção ou I para projecão), $a$ é o intercepto, $B_{i}$ são os coeficientes da regressão e $X_{i}$ são, de fato, os valores normalizados das variáveis,, vide Tabela 3.

Para testar a equação obtida pelo modelo da Equação I, utilizaram-se os dados das corridas. Foram separadas as corridas com projeção e corridas sem projeção. Os resultados para $M_{p i}$ são mostrados na Tabela 4.

O ideal é encontrar o valor I para corridas com projeção e 0 para corridas sem projeção, 0 que não foi alcançado. A imprecisão pode estar diretamente ligada à subjetividade do valor real de $M_{p i}$, pois existem corridas em que houve projeção e o valor da variável foi anotado como 0 . Vale considerar, ainda, que a reta de regressão se ajusta em $65,70 \%$, ou seja, também há os valores que estão fora deste ajuste.

\section{CONCLUSÃO}

Foi possível identificar, a partir de análises multivariadas, as principais variáveis que influenciam a projeção de escória no BOF e ainda sugerir um modelo de previsão de projeção. As evidências apuradas no acompanhamento mostram indícios de que muitas das projeções não são detectadas pelo operador, portanto, a análise de dados pode estar comprometida devido à subjetividade dos dados em relação à projeção. As principais variáveis que estão associadas à projeção são: Si no gusa líquido (\%); peso de minério adicionado $(\mathrm{t})$; tempo de sopro de oxigênio $(\mathrm{min})$; e peso de cal calcítica adicionada ( $\mathrm{t}$ ). $\mathrm{O}$ modelo matemático de regressão múltipla não explica todas as ocorrências de projeção o que pode ser devido à imprecisão dos dados utilizados, havendo a possibilidade de não identificação de uma projeção realmente ocorrida.

\section{Agradecimentos}

À ArcelorMittal R\&D, à UFOP (REDEMAT), e à CAPES (Código de Financiamento 00I).

\section{REFERÊNCIAS}

I Rizzo E. Processos de refino primário dos aços nos convertedores a oxigênio. São Paulo: ABM; 2006. p. 6I-64. O Sopro de Oxigênio.

2 Fruehan R, Turkdogan E. The making, shaping and treating of steel. IOth ed. Pittsburgh: AISE Steel Foundation; 1998. p. 102. Fundamentals of Iron and Steelmaking.

3 Albani CB, Furtado HS, Oliveira JR. Estudo das causas de projeções de escória no convertedor LD. In: Associação Brasileira de Metalurgia, Materiais e Mineração. ABMWeek 2017; São Paulo, Brazil. São Paulo: ABM. p. 289-298.

4 Hewage AK. Numerical Simulation of Slag Foaming with Reaction Kinetics in Oxygen Steelmaking [thesis]. Melbourne, Australia: Faculty of Science, Engineering and Technology, Swinburne University of Technology; 2017.

5 Harada Y, Ishihara M, Saito N, Nakashima K. Impedance measurement of simulated foaming slag for evaluation of gas phase fraction. ISIJ International. 20I7;57(I0): I733-I74I. 
6 Remus R, Monsonet M, Roudier S, Sancho L. Best Available Techniques (BAT) reference document for iron and steel production. Seville: Joint Research Centre; 2013. p. 354-376. Basic Oxygen Steelmaking and Casting.

7 Bramming M. Avoiding slopping in top-blow BOS Vessels [thesis]. Lulea: Lulea University of Technology; 2010.

8 Walker DI, Kemeny FL, Jone JAT. Vessel slopping detection. USA: Aistech; 2005. p. 7I I-72I.

9 Carvalho D, Castro H, Demuner L, Viana M, Wojnar P, Formage R, Schulz T, Totti B. Blowing Process Challenges for High rate of Iron Ore Pellet Addition - Process Development and Results at TKCSA. In: Associação Brasileira de Metalurgia, Materiais e Mineração. ABM Week 2017; São Paulo, Brazil. São Paulo: ABM. p. 637-645.

10 NCSS Statistical Software. Principal components analysis. USA: NCSS; 2017 [acesso em 10 ago. 2017]. Disponivel em: https://ncss-wpengine.netdna-ssl.com/wp-content/themes/ncss/pdf/Procedures/NCSS/Principal_Components_ Analysis.pdf

I Hair J Jr, Black W, Babin B, Anderson R, Tatham R. Análise multivariada de dados. 6. ed. Porto Alegre: Bookman; 2009.

Recebido em: 28 Out. 2019

Aceito em: 6 Nov. 2019 